\title{
Calidad metodológica y características de las tesis de pregrado de psicología de una universidad privada del Perú
}

\author{
Methodological Quality and Characteristics of the \\ Undergraduate Psychology Theses of a Private University of \\ Peru
}

Oscar J. Mamani Benito* (D) Universidad Peruana Unión, Lima, Perú. ORCID: https://orcid.org/0000-0002-9818-2601

Recibido 22-06-18 Revisado 30-06-18 Aprobado 17-09-18 En línea 19-09-18

\section{*Correspondencia}

Email: oscar.mb@upeu.edu.pe

\section{Citar como:}

Mamani Benito, O. (2018). Calidad metodológica y características de las tesis de pregrado de Psicología de una universidad privada del Perú. Propósitos y Representaciones 6(2), 301-338. Doi: http://dx.doi.org/10.20511/ pyr2018.v6n2.224

(C) Universidad San Ignacio de Loyola, Vicerrectorado de Investigación, 2018

(cc) BY-NC-ND Este artículo se distribuye bajo licencia CC BY-NC-ND 4.0 Internacional (http://creativecommons.org/licenses/by-nc-nd/4.0/). 


\section{Resumen}

Se tuvo como objetivo determinar la calidad metodológica e identificar las características de las tesis de psicología aprobadas en el periodo 2014 -2017 en una universidad privada de Perú. Corresponde a un estudio descriptivobibliométrico, donde la población de estudio fueron 161 tesis aprobadas en el periodo 2014 - 2017, de las cuales se analizaron 149 archivos en extenso disponibles en el repositorio institucional. Se utilizó como instrumento el formato de calificación del informe final de trabajos de investigación creado en la Universidad Nacional de Trujillo. Los principales resultados indican que el $72.5 \%$ de las tesis fueron realizadas por dos autores, el $81.2 \%$ corresponde al tipo correlacional, el $66.4 \%$ se orienta a la línea de investigación de psicología clínica y de la salud, el $47 \%$ involucró población escolar en su mayoría adolescentes (69.1\%) y solo el 6\% de los trabajos lograron ser publicados; en cambio, respecto a la calidad metodológica se halló que la mayoría de tesis (83) evidenciaron una calidad buena, y respecto a sus componentes se encontraron puntuaciones bajas en antecedentes (.57), tamaño muestral (.58), análisis estadístico (.43), diseño de contrastación (.49) y discusión (.53). Se concluye que las tesis sustentadas en el periodo 2014 - 2017 se caracterizan por ser de diseño no experimental, involucran mayormente población de estudio escolar en edad adolescente y mantienen una calidad entre regular a buena, evidenciando deficiencias metodológicas sobre todo en el manejo estadístico.

Palabras clave: Calidad metodológica; Tesis; Producción científica; Psicología 


\section{Summary}

The objective was to determine the methodological quality and identify the characteristics of the psychology theses approved in the 2014-2017 period of a private university in Peru. Corresponds to a descriptive-bibliometric study, where the study population was 161 theses approved in the period 2014 2017, of which 149 extensive files available in the institutional repository were analyzed. The qualification format of the final report of research work created at the National University of Trujillo was used as an instrument. The main results indicate that $72.5 \%$ of the theses were carried out by two authors, $81.2 \%$ corresponded to the correlation type, $66.4 \%$ were oriented to the clinical and health psychology research line, $47 \%$ involved the school population in mostly adolescents $(69.1 \%)$ and only $6 \%$ of the works were published; On the other hand, regarding the methodological quality, it was found that the majority of the theses (83) showed a good quality, and with respect to its components, low scores were found in antecedents (.57), sample size (.58), statistical analysis (. 43), contrast design (.49) and discussion (.53). It is concluded that the theses supported in the period 2014- 2017 are characterized by being of non experimental design, they involve mostly school study population in adolescent age and maintain a quality between regular and good, evidencing methodological deficiencies in the statistical management.

Keywords: Methodological quality; Thesis; Scientific Production; Psychology 


\section{Introducción}

Las tesis de grado son el principal insumo para la producción científica estudiantil, no obstante, la realidad de los universitarios en el Perú demuestra que esta no viene siendo valorada como un factor que aporta a la generación de nuevo conocimiento (Atamari-Anahui, Roque-Roque, Robles-Mendoza, Nina Moreno, \& Falcón-Huancahuiri, 2015; Miyahira, 2015; Taype-Rondán, Carbajal-Castro, Arrunategui-Salas, \& Chambi-torres, 2012)descriptive. Materials: Pre-graduate theses approved during 2000-2009. Interventions: Permission was obtained to access theses at the University library. A search for separate theses was conducted utilizing Google Scholar to find dates of publication. Personal scientific production of both theses consultants and jurors was also researched. Results were tabulated in Microsoft Excel 2010 and presented descriptively. Main outcome measures: Theses publication, scientific production of tutors and juries. Results: During 2000-2009, 2667 students graduated, 74 theses were approved, and only two were published. Twenty-one out of 67 consultants (31.3\% sino más bien como solo un requisito que en opinión de Portocarrero (2014) es considerada una cumbre a la que el estudiante muchas veces no sabe cómo llegar, generando así una actitud de querer realizarla de la manera más sencilla posible con tal de culminar el proceso y lograr la sustentación en un tiempo breve.

Aunque esta problemática se ha venido contemplando desde los comienzos del ejercicio de la investigación científica en las universidades, la situación en el Perú en opinión de San Martin y Garcia (2006) se hizo critica a partir del año 1991 cuando se expidió el decreto legislativo $\mathrm{N}^{\circ} 739$ donde la presentación y aprobación de una tesis para obtener el título profesional quedó relegada a ser solo una de tres opciones para la titulación, siendo este, el contexto donde la mayoría de estudiantes optaba por los cursos de actualización que la propia universidad gestionaba como alternativa según Ley. Posteriormente, casi después de una década las repercusiones no se harían esperar, pues la producción de conocimientos denotaba una crisis a tal 
punto que el Perú fue considerado como el País con la más baja proyección de producción científica en el año 2015 (Moya-Anegón et al., 2014).

Ante tal situación, el estado se ha visto presionado para formular estrategias con motivo de cambiar dicha realidad, una de ellas sería el hecho de volver a consignar en la nueva Ley universitaria la importancia debida a la tesis, colocándola nuevamente como requisito indispensable tanto para obtener el grado de bachiller como optar un título profesional, esto, claramente con la finalidad de impulsar nuevamente la producción científica estudiantil y tal como lo refiere León (2016) en el contexto universitario actual, que la tesis denote tres significados: una contribución al nuevo conocimiento, una oportunidad de aprendizaje respecto a cómo se investiga, y la certificación de competencias que la universidad confiere, con ello, se estaría generando un nuevo enfoque para el desarrollo de las investigaciones en el pregrado, pues por mucho tiempo las tesis en el Perú solo respondían a una orientación más profesionalizante que de investigación científica (Diaz \& Sime, 2016).

En el ámbito de la psicología y la salud mental, aunque son pocos los estudios que analizan la producción de nuevos conocimientos y calidad de las tesis en el Perú (Arias Gallegos, Ceballos Canaza, \& Arpasi Catacora, 2015; León, 2016; Luna-Solís, 2015; Uribe, Márquez, Amador, \& Chavez, 2011; Vera-Villaroel, Lopez-Lopez, Lillo, \& Silva, 2011)) la realidad no estaría muy alejada de las investigaciones en otras ramas de la ciencia como es el caso de las facultades de medicina, donde por ejemplo Taype-Rondán et al. (2012) al analizar las tesis de pregrado aprobadas entre el 2000 al 2009 en una universidad de Lima encontraron una proporción baja de tesis publicadas en revistas científicas, de igual manera Atamari-Anahui et al. (2015) en un estudio similar, esta vez en una universidad de provincia, luego de analizar la totalidad de las tesis aprobadas entre el 2000 y el 2012 hallaron que solo el 5\% de 398 tesis fueron publicadas. Siguiendo la misma línea, se pueden apreciar otros estudios relacionados a la calidad metodológica de las tesis como el de Mandujano-Romero y Grajeda (2013) quienes al analizar 172 tesis aprobadas en una universidad del Cusco hallaron que solo una 
de cada 5 fue aceptable, resaltando también las deficiencias significativas respecto al uso de referencias e instrumentos de medición; sin embargo también se hallaron otros estudios recientes como el de Zavaleta-Reyes y Tresierra-Ayala (2017) quienes luego de analizar 837 tesis aprobadas entre los años 2005 al 2014 en una universidad en Trujillo encontraron que en los últimos 10 años los trabajos de grado evidencian buena calidad y existe una tendencia a la mejora; finalmente, un estudio realizado por CastroMaldonado, Callirgos-Lozada, Caicedo-Písfil, Plasencia-Dueñas, y DíazVélez (2015) quienes luego de analizar 221 tesis en una universidad pública de Lima hallaron que la predominancia de los trabajos de grado se inclinan a ser descriptivas, basadas en literatura no actualizada y no acordes con las prioridades de salud, además, corroborando el estudio de Cusco hallaron que la publicación fue baja.

En este punto, es necesario también resaltar la situación de la producción científica en salud mental en el Perú, en ese sentido, los informes de unos pocos estudios como el de Luna-Solís (2015) quien analizo revistas indizadas en SCOPUS durante el año 2011 - 2013, demostraron que el Perú solo había producido 26 artículos que representan el $0.06 \%$ de la producción científica a nivel mundial, concluyendo que este indicador implica escaces, lo cual, corrobora lo indicado por Vera-Villaroel, Lopez-Lopez, Lillo, y Silva (2011) quienes concluyeron que Brasil, México, Argentina, Chile y Colombia demostraban mejores indicadores en términos de producción e impacto.

Es por ello que la formación de los investigadores desde el pregrado viene siendo una gran preocupación para la comunidad científica, puesto que Latinoamérica es la región con menor producción científica del mundo (Corrales-Reyes \& Dorta-Contreras, 2018); situación que es para nada ajena en el contexto peruano, sobre todo en el ámbito universitario donde se hace necesario evaluar la calidad metodológica de los trabajos de grado así como describir sus principales tendencias con el objetivo de analizar la orientación de la producción científica estudiantil; en ese sentido, el objetivo de la presente investigación es determinar la calidad metodológica y describir 
las características de las tesis de psicología sustentadas en una universidad privada de Lima.

\section{Método}

\section{Diseño de investigación.}

Se realizó un estudio descriptivo-bibliométrico de diseño no experimental.

\section{Población.}

La población de estudio fueron 161 tesis de pregrado de una Escuela Académico Profesional de Psicología aprobadas entre los años 2014 a 2017, las mismas se encuentran disponibles en el repositorio institucional perteneciente a universidad privada de Lima. Por motivos y restricciones de los autores se analizaron 149 trabajos de grado en archivo extensos; por lo tanto, se prescindió de realizar calculo alguno para hallar una muestra representativa pues la población analizada fue accesible y alcanzable.

\section{Instrumentos.}

Para evaluar la calidad metodológica de la tesis se recurrió al formato de calificación del informe final de trabajos de investigación creado en la Universidad Nacional de Trujillo y utilizado en un estudio similar por Zavaleta-Reyes y Tresierra-Ayala (2017), el mismo que evalúa la correcta utilización del método científico para la generación de conocimiento por estudio de la realidad. Dicho instrumento consta de 15 ítems distribuidos en 6 componentes las cuales evalúan: generalidades, plan de investigación, resultados, discusión, conclusiones, referencias bibliográficas y el resumen.

Respecto a su validez y confiabilidad, para cumplir con los objetivos de la presente investigación se validó el instrumento mediante el juicio de cinco expertos con experiencia en enseñanza de la metodología de la investigación 
y autores de artículos científicos en revistas indexadas de la especialidad. El promedio de evaluaciones fué hallado mediante un índice de acuerdo cuyo valor fue de .973 que indica una validez óptima. Una vez confirmada la validez de contenido se pasó a realizar una prueba piloto donde se aplicó el instrumento a una población de 30 tesis de una universidad privada de la ciudad de Juliaca, hallándose un indicador de consistencia interna de .830 según el Apha de Cronbach, lo cual evidencia una confiabilidad adecuada.

\section{Procedimiento.}

Una vez obtenido acceso a los trabajos en extenso, se procedió a recabar las siguientes características: número de autores, sede, año de presentación, tipo de estudio, tipo de población, grupo etareo, línea de investigación y publicación. Respecto a este último punto, se realizó una búsqueda en Google académico utilizando el algoritmo propuesto por Mayta-Tristán y Mezones-Holguín (2009) el mismo que consiste en redactar el titulo competo en el buscador de google académico, si no hubiera coincidencias entonces redactar las tres palabras claves en combinación con los apellidos de los autores, finalmente de no obtener resultados repetir la búsqueda en inglés.

Para determinar la calidad metodológica, los puntajes fueron categorizados en 5 rangos de 3 puntos cada uno quedando de la siguiente forma: Calidad muy buena (13-15), buena (10 - 12), regular (7 - 9), mala (4 6) y muy mala (1 - 3). Posteriormente, se pasó a analizar el contenido de cada uno de los trabajos evaluando sus principales subcomponentes como son: titulo (el cual debe informar adecuadamente el contenido de la investigación y mencionar las variables del problema), tipo y diseño (en el que se debe identificar adecuadamente el tipo de investigación con base en el objetivo y diseño de contrastación), antecedentes (que implica consignar antecedentes en base a la realidad problemática), justificación (en el que se debe explicar con precisión el valor teórico, social y metodológico del estudio), problema (planteado como una interrogante utilizando términos claros con base en la realidad problemática), objetivos (el cual debe responder a una sola 
pregunta y diferenciarse del resto de objetivos secundarios), hipótesis (el cual debe ser explicito con sustento científico y guardar coherencia con el problema planteado), diseño de contrastación (donde se evalúa si se aplica los procedimientos necesarios para verificar la hipótesis o resolver el problema), tamaño muestral (donde se evalúa si se especifica claramente la fórmula utilizada o los procedimientos que permiten reproducir todos los cálculos para hallar la muestra), análisis estadísticos (donde se evalúa si los estadísticos son los más adecuados para el contraste de las hipótesis propuestas), resultados (donde se evalúa si la interpretación se enfoca en describir los valores hallados sin recurrir a comentarios personales), discusión (donde se evalúa si la explicación de los resultados se centra en la aceptación o rechazo de las hipótesis planteadas), conclusiones (donde se evalúa si estas fluyen lógicamente de los resultados obtenidos y guardan coherencia con los objetivos planteados), referencias bibliográficas (donde se evalúa si estas son consistentes con el plan de investigación y de acuerdo con normas APA actualizadas), finalmente el resumen (el cual debe estructurado, concreto y coherente con el texto general de la tesis)

Respecto a la calificación, esta se basa en un máximo 15 puntos, por lo tanto según los cálculos se pudo determinar que un puntaje de 9.75 equivale a 13 en la escala vigesimal, lo cual se establece como nota mínima aprobatoria.

\section{Resultados}

Se hizo los análisis descriptivos de las principales características de las tesis de Psicología de la universidad en cuestión, correspondientes al periodo 2014 - 2017. De un total de 161 trabajos de grado, se pudieron analizar 149 archivo en extenso, la diferencia (12 trabajos) no pudo ser evaluada por restricciones de los autores quienes solo autorizaron mostrar la caratula y el resumen de su tesis.

Los resultados consignados en la tabla 1 muestran que el 59.7\% de los trabajos aprobados pertenecen a la sede principal ubicada en la ciudad de 
Lima, el $15.4 \%$ corresponden a otra sede ubicada en Tarapoto y un $24.8 \%$ a la sede ubicada en la ciudad de Juliaca. Del total de las tesis, el $49 \%$ fueron ejecutadas en el año 2016, 32.2\% en el año 2015, 11.4\% en el año 2017 y el $7.4 \%$ en el año 2014. Respecto al número de autores, se observa que el $72.5 \%$ de trabajos tuvieron dos autores y solo un $27.5 \%$ un solo autor, así también, respecto al tipo de investigación frecuente el $81.2 \%$ corresponde a estudios correlacionales, el $14.8 \%$ a estudios descriptivos, el $2.7 \%$ a estudios psicométricos y solo el 1.3\% a estudios que implican intervención. En cuanto a la correspondencia con líneas de investigación, el $66.4 \%$ se orienta a la psicología clínica y de la salud, el $20.1 \%$ a psicología positiva, el $4.7 \%$ a psicología educativa al igual organizacional (4.7\%), el $2.7 \%$ a psicometría y solo el $1.3 \%$ a social comunitaria.

Seguidamente, respecto al tipo de población, se observa que el $47 \%$ de los estudios involucró población escolar, el 20.8\% universitaria, el 12.1\% comunitaria, el $8.7 \%$ hospitalaria, y en porcentajes menores penitenciario (3.4\%), eclesiástico (3.4\%), centros de salud (2.7\%) y otros (2\%), así mismo, en cuanto al grupo etareo, el $69.1 \%$ de los estudios involucra adolescentes, el $18.1 \%$ adultos, el $11.4 \%$ adultos mayores y solo el $1.3 \%$ a niños. Finalmente, respecto a la tasa publicación de las tesis, se observa que solo $6 \%$ (9 tesis) lograron ser publicadas en revistas científicas indizadas, mientras que el 94\% es decir 140 aun no. 


\section{Tabla 1.}

Características de las tesis de psicología de una universidad privada de Perú. Periodo 2014 - 2017.

\begin{tabular}{|c|c|c|c|}
\hline & & $\mathrm{N}$ & $\%$ \\
\hline \multirow[t]{3}{*}{ Sede } & Lima & 89 & 59,7 \\
\hline & Tarapoto & 23 & 15,4 \\
\hline & Juliaca & 37 & 24,8 \\
\hline \multirow[t]{4}{*}{ Año de ejecución } & 2014 & 11 & 7.4 \\
\hline & 2015 & 48 & 32.2 \\
\hline & 2016 & 73 & 49.0 \\
\hline & 2017 & 17 & 11.4 \\
\hline \multirow[t]{2}{*}{ Numero de autores } & 1 & 41 & 27,5 \\
\hline & 2 & 108 & 72,5 \\
\hline \multirow[t]{4}{*}{ Tipo de estudio } & Descriptivo & 22 & 14.8 \\
\hline & Correlacional & 121 & 81.2 \\
\hline & Intervención & 2 & 1.3 \\
\hline & Psicométrico & 4 & 2.7 \\
\hline \multirow[t]{6}{*}{ Línea de investigación } & Psicología positiva & 30 & 20.1 \\
\hline & Clínica y de la salud & 99 & 66.4 \\
\hline & Educativa & 7 & 4.7 \\
\hline & Social y comunitaria & 2 & 1.3 \\
\hline & Organizacional & 7 & 4.7 \\
\hline & Psicometría & 4 & 2.7 \\
\hline \multirow[t]{8}{*}{ Tipo de población } & Hospitalario & 13 & 8.7 \\
\hline & Centro de salud & 4 & 2.7 \\
\hline & Comunidad & 18 & 12.1 \\
\hline & Escolar & 70 & 47.0 \\
\hline & Universitario & 31 & 20.8 \\
\hline & Penitenciario & 5 & 3.4 \\
\hline & Eclesiástico & 5 & 3.4 \\
\hline & Otros & 3 & 2.0 \\
\hline
\end{tabular}




\begin{tabular}{llcc}
\hline Grupo etario & Niño & 2 & 1.3 \\
& Adolescente & 103 & 69.1 \\
& Adulto & 27 & 18.1 \\
Publicación & Adulto mayor & 17 & 11.4 \\
& $\mathrm{Si}$ & 9 & 6.0 \\
& $\mathrm{No}$ & 140 & 94.0 \\
\hline
\end{tabular}

En cuanto a la calidad metodológica de las tesis analizadas, la tabla 2 resume de manera general los principales indicadores según año de aprobación, la misma, evidencia que 83 trabajos de grado obtuvieron una calificación en categoría buena, 51 en categoría regular, 13 en categoría muy buena y solo 2 en categoría mala. Cabe resaltar que ninguna evidenció una categoría muy mala.

\section{Tabla 2.}

Calidad de las tesis de psicología de una universidad privada de Perú. Periodo 2014 - 2017.

\begin{tabular}{llllll}
\hline & 2014 & 2015 & 2016 & 2017 & Total \\
\hline Mala & 1 & 0 & 1 & 0 & 2 \\
Regular & 3 & 14 & 31 & 3 & 51 \\
Buena & 6 & 33 & 33 & 11 & 83 \\
Muy buena & 1 & 1 & 8 & 3 & 13 \\
Total & 11 & 48 & 73 & 17 & 149 \\
\hline
\end{tabular}

En el mismo contexto, la tabla 3 muestra a profundidad el análisis de la evaluación de los principales componentes en los trabajos de grado aprobados en el periodo 2014 - 2017. En general, se observa que en promedio las tesis evidencian un puntaje de 10.4 que en una escala vigesimal corresponde a 13.9, que a su vez demuestra una valoración aprobatoria; además, al analizar los promedios anuales se puede observar que existe una tendencia a mejorar con el transcurso del tiempo $(2014=13.69 ; 2015=13.6 ; 2016=13.28$ y 2017 $=15.01)$. Respecto a los componentes, los ítems con mayores puntuaciones promedio son: titulo (.96), problema (.87), objetivos (.80), resultados (.87) $\mathrm{y}$ conclusiones (.80); mientras que se hallaron puntuaciones bajas en los 
ítems antecedentes, (.57) tamaño muestral (.58), análisis estadístico (.43), diseño de contrastación (.49) y discusión (.53), lo cual indica deficiencias metodológicas en estos apartados.

\section{Tabla 3.}

Calificación del trabajo de tesis de psicología por año. Periodo 2014 - 2017

\begin{tabular}{lccccc}
\hline \multicolumn{1}{c}{ Año } & $\mathbf{2 0 1 4}$ & $\mathbf{2 0 1 5}$ & $\mathbf{2 0 1 6}$ & $\mathbf{2 0 1 7}$ & Promedio \\
\hline Titulo & 0.95 & 0.98 & 0.97 & 0.94 & 0.96 \\
Resumen & 0.86 & 0.94 & 0.83 & 0.85 & 0.87 \\
Problema & 0.68 & 0.81 & 0.84 & 0.85 & 0.8 \\
Objetivos & 0.73 & 0.81 & 0.86 & 0.85 & 0.81 \\
Justificación & 0.64 & 0.57 & 0.66 & 0.65 & 0.63 \\
Antecedentes & 0.50 & 0.50 & 0.53 & 0.74 & 0.57 \\
Tipo de investigación & 0.64 & 0.64 & 0.60 & 0.94 & 0.71 \\
Tamaño muestral & 0.68 & 0.59 & 0.51 & 0.53 & 0.58 \\
Hipótesis & 0.55 & 0.54 & 0.58 & 0.82 & 0.62 \\
Análisis estadístico & 0.64 & 0.47 & 0.32 & 0.29 & 0.43 \\
Resultados & 0.95 & 0.95 & 0.82 & 0.76 & 0.87 \\
Diseño de contrastación & 0.55 & 0.44 & 0.27 & 0.71 & 0.49 \\
Discusión & 0.50 & 0.50 & 0.51 & 0.62 & 0.53 \\
Conclusiones & 0.68 & 0.78 & 0.84 & 0.88 & 0.8 \\
Referencias bibliográficas & 0.73 & 0.68 & 0.84 & 0.82 & 0.77 \\
Total & 10.27 & 10.20 & 9.96 & 11.26 & 10.4 \\
Escala vigesimal & 13.69 & 13.6 & 13.28 & 15.01 & 13.9 \\
Total informes & 11 & 48 & 73 & 17 & 149 \\
\hline
\end{tabular}

\section{Discusión}

Se ha encontrado que el $72.5 \%$ de las tesis fueron realizadas por dos autores lo cual significa que los estudiantes perciben que su elaboración sería más factible cuando tienen el apoyo de un compañero, así mismo, el hecho de que el $81.2 \%$ de investigaciones obedezca al tipo correlacional y solo $1.3 \%$ a intervenciones estaría relacionada a la actitud que muestran los tesistas (Mamani, 2015) pues la prioridad al encarar el trabajo de grado sería tan solo lograr la sustentación en un tiempo breve, más que la motivación de 
contribuir a la generación de nuevo conocimiento. Por otro lado, el hecho de que el $66.4 \%$ de investigaciones se haya orientado bajo una línea de investigación de psicología clínica y de la salud y el indicativo de que el $47 \%$ haya tomado en cuenta una población escolar - adolescente (69.1\%) obedecería a circunstancias ligadas al lugar de realización de prácticas profesionales e internado de los tesistas quienes generalmente ya en sus últimos ciclos académicos procuran presentar y ejecutar sus trabajos de grado, aunado a esto, considerar que dicha universidad mantiene convenios principalmente con Instituciones educativas como colegios e institutos, así como del sector salud generando alianzas con hospitales y centros de salud, los cuales, serian tomados en cuenta como primera opción por parte de los tesistas para plantear y ejecutar sus proyectos de tesis.

Otro de los hallazgos, que en realidad no llama la atención sino más bien corrobora lo existente en los reportes de la literatura científica tiene que ver con el indicador respecto a la publicación de tesis, el mismo que evidencia que solo un $6 \%$ de los trabajos fueron publicados en alguna revista científica indexada de especialidad. Este resultado es similar a los informados por Castro-Maldonado et al. (2015)are a way of doing research. Previous studies show a thesis published between $2.7 \%$ to $17.6 \%$ in indexed journals. Objective: To describe the characteristics of the undergraduate thesis of a medical school. Material and Methods: Bibliometric study. 221 theses were reviewed, collecting: year, number of authors, advisers, and references, study population, and national priorities for health research. A search was performed on Google Scholar to assess publication. Descriptive statistics were used. Results: Of the total, $91.6 \%$ of thesis had one advisor, $76 \%$ were descriptive, $82.8 \%$ were done in a hospital population, $62.4 \%$ in adults. A lower production was found in the years 2008-2010. Of the theses between $2010-2014,72.9 \%$ did not correspond to any national health research priority. Only $6.8 \%$ was based on literature of the last five years. Only 9 (4.1\% quienes luego de analizar 221 tesis hallaron que solo el $4.1 \%$ fueron publicadas, así también con el estudio de Taype-Rondán et al. (2012) quienes luego de 
analizar 74 tesis de pregrado encontraron que solo dos fueron publicadas, finalmente con el de Atamari-Anahui et al. (2015) quienes reportaron publicaciones de solo el 5\% de 398 trabajos de grado. Una explicación ante esta realidad puede hallarse al comprender la dinámica del proceso de asesoría entre el estudiante y el asesor, por un lado son pocos los estudiantes que demuestran intenciones de realizar investigaciones con el fin de publicar (Toro-Huamanchumo, Failoc-Rojas, \& Díaz-Vélez, 2015) y por otro lado el rol de los asesores de tesis no sería el esperado pues en vez de impulsar la realización de trabajos con impacto social y generar publicaciones para impulsar la producción científica estudiantil, este tan solo estaría orientado a cumplir una responsabilidad que solo llega hasta la sustentación del trabajo (Atamari-Anahui, Sucasaca-Rodríguez, \& Marroquin-Santa Cruz, 2016; Oyola-García, 2015).

Pasando a otro punto importante que tiene que ver con la calidad metodológica de las tesis, los hallazgos en el presente estudio demuestran que más de la mitad de los trabajos analizados demuestra una calidad buena (83) y que otra gran parte (51) evidencia una calidad regular. Estos resultados son similares a los hallazgos de Zavaleta-Reyes y Tresierra-Ayala (2017) quienes luego de analizar 837 trabajos de grado en una facultad de medicina encontraron que el $60.3 \%$ demuestran buena calidad y el $22.3 \%$ regular calidad, sin embargo, son contrarios a los hallados por Mandujano-Romero y Grajeda (2013) quienes luego de analizar 284 tesis para optar el título de Médico Cirujano encontraron que solo el 20.3\% demostraba una calidad aceptable; al respecto, quizá una explicación ante esta diferencia puede encontrarse claramente al descubrir que las políticas de investigación son distintas en cada universidad y tomando en cuenta la línea del conocimiento existen claras diferencias al momento de ejercer y evaluar la investigación científica tanto en las facultades de Medicina como en las de Psicología.

Continuando con el análisis, al profundizar en los principales componentes de los trabajos de grado analizados, se descubrió deficiencias metodológicas en el desarrollo de los antecedentes (.57) los cuales no evidencian una relación 
con la realidad problemática, así también, al precisar el tamaño muestral (.58) no se especifican adecuadamente los criterios utilizados lo cual no permite reproducir los cálculos realizados, de igual manera, al argumentar el análisis estadístico empleado (.43) no se precisa con total objetividad los procedimientos para el contraste de hipótesis (análisis estadístico $=.43$ ), lo cual también influye en los resultados pues no se visualiza el procedimiento de prueba de hipótesis (diseño de contrastación $=.49$ ), finalmente, en el tema de la discusión se halló que el enfoque de la redacción se orienta a una interpretación general no consignando una argumentación en cuestión de la aceptación o rechazo de las hipótesis planteadas (discusión $=.53$ ). Estos resultados, difieren de los hallazgos encontrados por Mandujano-Romero y Grajeda (2013) quienes al analizar 284 tesis de medicina encontraron deficiencias metodológicas en aspectos como: referencias bibliográficas, unidad de estudio y operacionalización de variables; así también, diferencias respecto al trabajo de Zavaleta-Reyes y Tresierra-Ayala (2017) quienes luego de analizar 885 trabajos de grado descubrieron deficiencias en los componentes de: hipótesis, conclusiones, resumen y justificación. Quizá una explicación a estas diferencias podría acentuarse al identificar que la población de estudio en estas investigaciones difiere completamente a la del presente estudio.

En conclusión, a pesar de la necesidad de investigaciones científicas en Psicología para dar respuesta a los diferentes problemas psicosociales del país (Aiquipa, Ramos, Curay, \& Guizaldo, 2018), como son la violencia, el feminicidio, la corrupción, entre otros problemas latentes, es visible que los estudios planteados desde la universidad en estas áreas son escasas y aun los que se plantean evidencian serias limitaciones en su calidad metodológica y corresponden a estudios básicos que demuestran la poca intención de realizar trabajos de mayor impacto social como son los experimentales, todo esto, 
limita significativamente no solo la producción científica estudiantil del país sino también el proceso de formación de nuevos investigadores para el Perú.

\section{Referencias}

Aiquipa, J., Ramos, C., Curay, R., \& Guizaldo, L. (2018). Factores implicados para realizar o no realizar tesis en estudiantes de psicología. Revista Propositos y Representaciones, 6(1), 2-26. Doi: https://doi. org/10.20511/pyr2018.v6n1.180

Arias Gallegos, W., Ceballos Canaza, K., \& Arpasi Catacora, S. (2015). El aporte de los psicólogos peruanas en la Revista Latinoamericana de Psicologia de 1994 al 2014: un estudio bibliometrico. Revista Peruana de Psicologia y Trabajo Social, 4(1), 57-76. Recuperado de: http:// revistas.uigv.edu.pe/index.php/psicologia/article/view/127

Atamari-Anahui, N., Roque-Roque, J. S., Robles-Mendoza, R. A., Nina Moreno, P. I., \& Falcón-Huancahuiri, B. M. (2015). Publicación de tesis de pregrado en una facultad de Medicina en Cusco, Perú. Revista Medica Herediana, 26(4), 217-221. Doi: https://doi.org/10.20453/rmh. v26i4.2707

Atamari-Anahui, N., Sucasaca-Rodríguez, C., \& Marroquin-Santa Cruz, J. A. (2016). Publicación científica de asesores de tesis de pregrado en una escuela de medicina de Cusco, Perú. Investigación en Educación Médica, 5(20), 279-280. Doi: https://doi.org/10.1016/j.riem.2016.05.002

Castro-Maldonado, B., Callirgos-Lozada, C., Caicedo-Písfil, M., PlasenciaDueñas, E., \& Díaz-Vélez, C. (2015). Características de las tesis de pre-grado de Medicina de una universidad pública del Perú. Horizonte Medico, 15(3), 34-39. Recuperado de: http://www.horizontemedicina. usmp.edu.pe/index.php/horizontemed/article/view/298

Diaz, C., \& Sime, L. (2016). Las tesis de doctorado en educación en el Perú : Un perfil de la producción académica en el campo educativo. Revista Peruana de Investigacion Educativa, (8), 5-40. Recuperado de http:// www.siep.org.pe/wp-content/uploads/Artículo-1.pdf 
León, F. (2016). ¿Qué significa una tesis de investigación en psicología en el Perú? Revista Persona, (19), 151-166. Recuperado de: https://revistas. ulima.edu.pe/index.php/Persona/article/view/977

Luna-Solís, Y. (2015). Producción científica en salud mental en Perú. Reto en tiempos de reforma de salud. Acta Médica Peruana, 32(1), 36-40. Recuperado de: http://www.amp.cmp.org.pe/index.php/AMP/article/ view/171

Mamani Benito, O. J. (2015). Actitud hacia la investigación y su importancia en la elección de la modalidad de tesis para optar el título profesional. Revista Científica de Ciencias de La Salud, 4(1), 22-27. Doi: https://doi. org/10.17162/rccs.v4i1.158

Mandujano-Romero, E., \& Grajeda, P. (2013). Calidad de las tesis para obtener el título de médico cirujano, Universidad Nacional de San Antonio Abad del Cusco - Perú, 2000 - 2009. Acta Médica Peruana, 30(2), 70-74. Recuperado de: http://www.scielo.org.pe/scielo.php?script=sci_arttext \&pid $=$ S1728-59172013000200004

Mayta-Tristán, P., \& Mezones-Holguín, E. (2009). Aclaración editorial. Revista Peruana de Medicina Experimental y Salud Pública, 26(3), 411-412. Doi: http://dx.doi.org/10.17843/rpmesp.2009.263.1398

Miyahira, J. (2015). ¿Por qué la tasa de publicación de las tesis en Medicina es baja?: Posibles explicaciones. Revista Medica Herediana, 26(4), 207208. Doi: https://doi.org/10.20453/rmh.v26i4.2698

Moya-Anegón, F., Bustos-González, A., Chinchilla-Rodríguez, Z., Corera-Álvarez, E., López-Illescas, C., \& Vargas-Quesada, B. (2014). Principales indicadores bibliométricos de la actividad cientifica peruana, 2006-2011. Recuperado de: http://bvcyt.concytec.gob.pe/ images/publicaciones/principales_indicadores_2006_2011.pdf

Oyola-García, A. E. (2015). El asesor de tesis. Acta Médica Peruana, 32(2), 131-132. Recuperado de: http://www.amp.cmp.org.pe/index.php/AMP/ article/view/147

San Martin, F., \& Garcia, M. (2006). La tesis y su problemática en la Facultad de Medicina Veterinaria de la UNMSM. Revista de Investigaciones 
Veterinarias del Perú, 17(1), 81-88. Doi: http://dx.doi.org/10.15381/ rivep.v17i1.1464

Taype-Rondán, Á., Carbajal-Castro, C., Arrunategui-Salas, G., \& Chambitorres, J. (2012). Limitada publicación de tesis de pregrado en una facultad de medicina de Lima, Perú, 2000-2009. Anales de la Facultad de Medicina, 73(6), 153-157. Doi: https://doi.org/10.15381/anales. v73i2.858

Toro-Huamanchumo, C. J., Failoc-Rojas, V. E., \& Díaz-Vélez, C. (2015). Participación en sociedades científicas estudiantiles y en cursos extracurriculares de investigación, asociados a la producción científica de estudiantes de medicina humana: estudio preliminar. FEM: Revista de la Fundación Educación Médica, 18(4), 293-298. Doi: https://doi. org/10.4321/S2014-98322015000500011

Uribe, J. I., Márquez, C., Amador, G., \& Chavez, A. (2011). Percepción de la investigación científica e intención de elaborar tesis en estudiantes de psicología y enfermería. Enseñanza e Investigación en Psicología, 16(1), 15-26. Recuperado de http://www.redalyc.org/pdf/292/29215963002. pdf

Vera-Villaroel, P., Lopez-Lopez, W., Lillo, S., \& Silva, L. M. (2011). La producción científica en psicología latinoamericana : Un análisis de la investigación por países. Revista Latinoamericana de Psicología., 43(1), 95-104. Doi: http://dx.doi.org/10.14349/rlp.v43i1.613

Zavaleta-Reyes, C., \& Tresierra-Ayala, M. (2017). Calidad metodológica del trabajo de grado de bachiller en una facultad de medicina. Educación Medica, 18(4), 233-241. Doi: https://doi.org/10.1016/j. edumed.2016.06.020 